with some evidence of minor in-host evolution, consistent with prolonged infection rather than alternative lineage reinfection.

Serology tests were performed on October 14 and November 16. SARS-CoV-2 IgA, IgG, and total antibody were not detected (Table 1). The patient was hypogammaglobulinemic throughout.

While in the hospital, the patient received 3 units of ABO-compatible convalescent plasma through compassionate access on October 16 and 17. He did not receive remdesivir, dexamethasone, or antibiotics. Cough and shortness of breath resolved, and he was discharged home on October 18. The patient was required to isolate at home, and subsequent swabs detected SARS-CoV-2 on PCR until November 16, when his test result was "not detected" for the first time (Table 1). Swabs following discharge have not demonstrated culturable virus.

This is an unusual case of SARS-CoV-2 infection with late symptom onset (72 days after initial diagnosis) in an immunocompromised patient. Possible triggers for this include bsAb therapy, cytokine release syndrome, or dexamethasone use. The former 2 factors are unlikely given the very low dose of bsAb and the lower $\mathrm{Ct}$ values for SARS-CoV-2 PCR and culturable virus.

The virus was still culturable, which raised concerns about transmissibility. The patient continued (until November 16, 111 days) to have prolonged PCR positivity with no development of antibodies to SARS-CoV-2 and did not meet hospital or statewide guidelines for clearance.

Given the transition to elimination of sustained community transmission of COVID-19 in Victoria, a very cautious approach was taken to the isolation and public health management of this case. The fact that there was no evidence that this case infected any close household or other contacts over an extended period raises the question of whether prolonged infection, even with culturable virus, poses an ongoing infection risk to others, and whether prolonged isolation with potential significant negative psychological effects is justified. ${ }^{9}$ More research into this important issue is needed.

Acknowledgments. We thank the patient and his family for their consent and participation in this report. We thank James Daly (Australian Red Cross
Lifeblood) for his assistance with the provision of convalescent plasma for the patient.

Financial support. No financial support was provided relevant to this article.

Conflicts of interest. G.P.G. is an advisory board member for Novartis, Roche, Gilead, Sandoz, and Janssen and has received research funding from Janssen, BeiGene, AstraZeneca, AbbVie, and MSD.

\section{References}

1. Wang C, Xu M, Zhang Z. A case of COVID-19 with long duration of viral shedding. J Microbiol Immunol Infect 2021;54:152-153.

2. Liu WD, Chang SY, Wang JT, et al. Prolonged virus shedding even after seroconversion in a patient with COVID-19. J Infect 2020;81:318-356.

3. Choi B, Choudhary MC, Regan J, et al. Persistence and evolution of SARS-CoV-2 in an immunocompromised host. N Engl J Med 2020 ;383: 2291-2293.

4. Helleberg M, Niemann CU, Moestrup KS, et al. Persistent COVID-19 in an immunocompromised patient temporarily responsive to two courses of remdesivir therapy. J Infect Dis 2020;222:1103-1107.

5. Victorian coronavirus (COVID-19) data. Department of Health and Human Services, Victorian Government website. https://www.dhhs.vic.gov.au/ victorian-coronavirus-covid-19-data. Published November 23, 2020. Accessed February 2, 2021.

6. Case and contact management guidelines, version 25. Department of Health and Human Services, Victorian Government website. https://www.dhhs.vic. gov.au/health-services-and-professionals-coronavirus-covid-19. Published October 14, 2020. Accessed February 2, 2021.

7. Communicable diseases network Australia national guidelines for public health units, version 3.10. Australian Government Department of Health website. https://www1.health.gov.au/internet/main/publishing.nsf/Content/ cdna-song-novel-coronavirus.ht. Published October 28, 2020. Accessed March 16, 2020.

8. Seemann T, Lane CR, Sherry NL, et al. Tracking the COVID-19 pandemic in Australia using genomics. Nat Commun 2020;11:4376.

9. Brooks SK, Webster RK, Smith LE, et al. The psychological impact of quarantine and how to reduce it: rapid review of the evidence. Lancet 2020;395:912-920.

\title{
Povidone-iodine preprocedural rinse-An evidence-based, second-line defense against severe acute respiratory coronavirus virus 2 (SARS-CoV-2) in dental healthcare
}

\author{
Raj Kumar Maurya BDS, MDS ${ }^{1}$ (1), Harpreet Singh BDS, MDS $^{2}$ (1), Pranav Kapoor BDS, MDS², Poonam Sharma BDS, MDS² \\ and Dhirendra Srivastava BDS, MDS, MOMS RCPS (Glasgow) ${ }^{2}$ \\ ${ }^{1}$ Central Government Dental Unit, Nagaland, India and ${ }^{2}$ Department of Orthodontics \& Dentofacial Orthopedics, ESIC Dental College \& Hospital, New Delhi
}

To the Editor-Ever since it began, coronavirus disease 2019 (COVID-19) pandemic has brought disruptions to almost all aspects of society, with far-reaching adverse impacts on clinical

\footnotetext{
Author for correspondence: Dr Harpreet Singh, E-mail: drhpreetesic@gmail.com Cite this article: Maurya RK, et al. (2022). Povidone-iodine preprocedural rinse-An evidence-based, second-line defense against severe acute respiratory coronavirus virus 2 (SARS-CoV-2) in dental healthcare. Infection Control \& Hospital Epidemiology, 43: 822-824, https://doi.org/10.1017/ice.2021.90
}

and economic fronts. Even amid effective vaccine rollout, which has rekindled the hope of ending this unprecedented global disaster, the COVID-19 pandemic continues unabated, with reports of a highly transmissible, mutant, severe acute respiratory coronavirus virus 2 (SARS-CoV-2) VOC 202012/01 strain and another SARS$\mathrm{CoV}-2$ 20H/501Y.V2 variant. These strains could drive even larger waves of disease. ${ }^{1}$ Due to high viral loads and the consequent transmission potential of asymptomatic and minimally symptomatic

(c) The Author(s), 2021. Published by Cambridge University Press on behalf of The Society for Healthcare Epidemiology of America. This is an Open Access article, distributed under the terms of the Creative Commons Attribution licence (http://creativecommons.org/licenses/by/4.0/), which permits unrestricted re-use, distribution, and reproduction in any medium, provided the original work is properly cited. 
patients, ${ }^{2}$ reducing the viral load in the oropharynx with adequate oral prophylactic measures is imperative to contain and prevent the spread of SARS-CoV-2 in public and dental healthcare settings. Considering the high-risk nature of the dental healthcare occupation due to the propensity for close-contact transmission through infective saliva, droplet splatter, and blood-mixed aerosols, procedural mitigation strategies involving prophylactic use of preprocedural mouth rinses (PPMRs) has been recommended to attenuate nosocomial transmission of SARS-CoV-2. ${ }^{3}$ Different oral antiseptic agents have been employed as a second layer of defence against microbial load in dental aerosols: povidone-iodine (PVP-I), chlorhexidine gluconate (CHX), hydrogen peroxide, cetylpyridinium chloride (CPC), and essential oils. ${ }^{4}$ A comparative in vitro study evaluating virucidal efficacy of different oral rinses demonstrated that mouth formulations containing 1\% PVP-I, combination of dequalinium chloride and benzalkonium chloride, and a combination of ethanol and essential oils can significantly reduce SARS-CoV-2 viral infectivity within short exposure times of 30 seconds. ${ }^{5}$ However, CHX-based mouth rinse exhibited weak virucidal efficacy. A recent systematic review found no scientific evidence supporting the virucidal activity of hydrogen peroxide mouthwash. ${ }^{6}$ With the growing evidence amid the COVID-19 pandemic, evidence-based recommendations in favor of PVP-I as PMR have been increasing.

PVP-I or iodopovidone has been ubiquitously used as an antiseptic in healthcare settings for decades. Comprising iodine and the water-soluble polymer polyvinylpyrrolidone, PVP-I is considered favorable for its slow and gradual iodine release, minimizing toxicity, and for the resultant viral inactivation arising from its oxidative effect and lipid shell-membrane destruction. ${ }^{7}$ Regarding the virucidal activity of PVP-I against SARS-CoV-2, Bidra et $\mathrm{al}^{8}$ were among the first researchers to provide direct evidence of rapid in vitro virucidal action of $0.5 \%$ PVP-I oral rinse at the lowest contact time of 15 seconds. These researchers also showed that $70 \%$ ethanol-based rinse requires a minimum contact time of 30 seconds rather than 15 seconds to completely inactivate SARS-CoV-2. Subsequent in vitro studies conducted by Hassandarvish et $\mathrm{al}^{9}$ demonstrated potent and rapid virucidal efficacy of $0.5 \%$ and $1 \%$ PVP-I rinse at the lowest contact time of 15 seconds, and Anderson et $\mathrm{al}^{10}$ also demonstrated the efficacy of $1.0 \% \mathrm{w} / \mathrm{v}$ PVP-I oral rinse and $0.45 \% \mathrm{w} / \mathrm{v}$ PVP-I throat spray with a contact time of 30 seconds.

In real-time hospital settings, Martinez et $\mathrm{al}^{11}$ reported significant reduction in SARS-CoV-2 viral load after rinsing with 1\% PVP-I for 1 minute, with 3-hourly sustained clinical effects. A recently published sole randomized controlled trial involving 36 SARS-CoV-2-positive patients evaluated the in vivo efficacy of 3 commercial mouth rinses compared with water: PVP-I, $\mathrm{CHX}$, and CPC. ${ }^{12}$ Rinsing with $0.075 \%$ CPC and $0.5 \%$ PVP-I for 30 seconds decreased the salivary SARS-CoV-2 levels within 5 minutes of use, and the subsequent effects were sustained at 6-hour time points.

No cytotoxic effects of 1\% PVP-I mouthwash have been reported when used at a concentration of $0.63 \mathrm{mg} / \mathrm{mL}$ (ie, 1:16 or lower dilution of the product). ${ }^{9}$ Moreover, in addition to a well-established safety profile, PVP-I also exhibits good tolerability at concentrations as high as $2.5 \%$ for up to 5 months, with the absence of tooth or tongue discoloration or taste disturbances. ${ }^{13}$ Unlike alcohol-based mouth rinse, it can also be used in conjunction with electrocautery, which also make it a suitable choice for use in maxillofacial surgery. Few contraindications for the use of PVP-I solutions include type 1 anaphylactic allergy to iodine (rarely encountered), active thyroid disease, pregnancy, and radioactive iodine therapy. Hence, proper medical and drug history should be reviewed before their use.

In view of the fluid situation posed by the ongoing pandemic, Kirk-Bailey et $\mathrm{al}^{14}$ recommend the use of $9 \mathrm{~mL} \mathrm{0.5 \%} \mathrm{PVP-I} \mathrm{as} \mathrm{a}$ mouthwash and $0.28-0.3 \mathrm{~mL} 0.5 \%$ PVP-I as a nasal spray solution in each nostril, both for the patient before examination and/or treatment, and for the clinical staff prior to patient contact (repeated every 2-3 hours, up to 4 times a day, if multiple patients are seen). Due to the chemical instability of PVP-I with respect to disproportionation into constituent equilibrium species, freshly prepared dilutions should be used, and they should be preserved in the refrigerator for subsequent patients through the day. ${ }^{8}$

While reconciling the fact that postpandemic resurgences may occur as late as $2024,{ }^{15}$ safeguarding the oral healthcare workforce and patients becomes even more pertinent considering the perceived vulnerability to the SARS-CoV-2 infection in dental settings. With a growing body of direct evidence consistently supporting the effectiveness of PVP-I against SARS-CoV-2, it would be reasonable to prioritize the use of PVP-I as an effective and adjunctive procedural risk mitigation measure in dental healthcare in the contemporary pandemic crisis and through unforeseen similar pandemic threats.

\section{Acknowledgments.}

Financial support. No financial support was provided relevant to this article.

Conflicts of interest. All authors report no conflicts of interest relevant to this article.

\section{References}

1. Davies NG, Barnard RC, Jarvis CI, et al. Estimated transmissibility and severity of novel SARS-CoV-2 variant of concern 202012/01 in England. medRxiv 2020. doi: 10.1101/2020.12.24.20248822.

2. Zou L, Ruan F, Huang M, et al. SARS-CoV-2 viral load in upper respiratory specimens of infected patients. N Engl J Med 2020;382:1177-1179.

3. Peng X, Xu X, Li Y, Cheng L, Zhou X, Ren B. Transmission routes of 2019-nCoV and controls in dental practice. Int J Oral Sci 2020;12:9.

4. Harrel SK, Molinari J. Aerosols and splatter in dentistry: a brief review of the literature and infection control implications. J Am Dent Assoc 2004; 135:429-437.

5. Meister TL, Brüggemann Y, Todt D, et al. Virucidal efficacy of different oral rinses against severe acute respiratory syndrome coronavirus 2.J Infect Dis 2020;222:1289-1292.

6. Ortega KL, Rech BO, El Haje GLC, Gallo CB, Pérez-Sayáns M, Braz-Silva $\mathrm{PH}$. Do hydrogen peroxide mouthwashes have a virucidal effect? A systematic review. J Hosp Infect 2020;106:657-662.

7. Nagatake T, Ahmed K, Oishi K. Prevention of respiratory infections by povidone-iodine gargle. Dermatology 2002;204 suppl 1:32-36.

8. Bidra AS, Pelletier JS, Westover JB, Frank S, Brown SM, Tessema B. Rapid in vitro inactivation of severe acute respiratory syndrome coronavirus 2 (SARS-CoV-2) using povidone-iodine oral antiseptic rinse. J Prosthodont 2020;29:529-533.

9. Hassandarvish $\mathrm{P}$, Tiong V, Mohamed NA, et al. In vitro virucidal activity of povidone iodine gargle and mouthwash against SARSCoV-2: implications for dental practice. Br Dent J 2020. doi: 10.1038/s41415-020-2402-0.

10. Anderson DE, Sivalingam V, Kang AEZ, et al. Povidone-iodine demonstrates rapid in vitro virucidal activity against SARS-CoV-2, the virus causing COVID-19 disease. Infect Dis Ther 2020;9:669-675.

11. Martínez Lamas L, Diz Dios P, Pérez Rodríguez MT, et al. Is povidone iodine mouthwash effective against SARS-CoV-2? First in vivo tests. Oral Dis 2020. doi: 10.1111/odi.13526. 
12. Seneviratne CJ, Balan P, Ko KKK, et al. Efficacy of commercial mouthrinses on SARS-CoV-2 viral load in saliva: randomized control trial in Singapore. Infection 2020. doi: 10.1007/s15010-020-01563-9.

13. Frank S, Capriotti J, Brown SM, Tessema B. Povidone-iodine use in sinonasal and oral cavities: a review of safety in the COVID-19 era. Ear Nose Throat J 2020;99:586-593.
14. Kirk-Bayley J, Combes J, Sunkaraneni V, Challacombe S. The use of povidone iodine nasal spray and mouthwash during the current COVID-19 pandemic may reduce cross infection and protect healthcare workers. SSRN 2020. doi: 10.2139/ssrn.3563092.

15. Kissler SM, Tedijanto C, Goldstein E, Grad YH, Lipsitch M. Projecting the transmission dynamics of SARS-CoV-2 through the postpandemic period. Science 2020;368:860-868.

\title{
Filtration evaluation of expired elastomeric P-100 filter cartridges after months of real-world use during the coronavirus disease 2019 (COVID-19) pandemic
}

\author{
Anthony W. Baffoe-Bonnie MD ${ }^{1,3}$ (1), Kermit Sheng Zhang BS ${ }^{1}$, Jin Pan MS², Kirsten Simpkins MPH, BSN, RN ${ }^{3}$ and \\ Linsey C. Marr PhD² \\ ${ }^{1}$ Virginia Tech Carilion School of Medicine, Roanoke, Virginia, ${ }^{2}$ Civil and Environmental Engineering, Virginia Tech, Blacksburg, Virginia and ${ }^{3}$ Carilion Clinic \\ Roanoke Memorial Hospital, Roanoke, Virginia
}

To the Editor-Complicating the frontline healthcare worker (HCW) response to the COVID-19 pandemic has been the widespread shortage of personal protective equipment (PPE), including disposable single-use N95 filtering facepiece respirators (FFRs). To that end, the Centers for Disease Control and Prevention (CDC) has provided guidance on strategies that healthcare facilities may use to conserve N95s. This guidance includes extended use and reuse of previously single-use N95 FFRs coupled with various modalities of decontamination and augmenting respirator supplies with reusable devices like elastomeric respirators. ${ }^{1,2}$

In the healthcare setting, there is consensus that elastomeric filter cartridges should be replaced if they become visibly soiled, wet, damaged, or notably harder to breathe through. ${ }^{2}$ Otherwise, the recommended timing for replacing elastomeric filter cartridges varies from every 30 days to annually. 3,4

We previously determined that unopened P100 elastomeric filter cartridges received from the strategic national stockpile (SNS), which were 6 years past their shelf-life, offered similar filtration efficiency to the N95 respirator. ${ }^{5}$ Our current study evaluates how these filters performed following use in the real-world (ie, healthcare) setting. Information pertaining to this topic is lacking.

Three pairs of P100 filter cartridges that had been in use for 5 and 6 months were obtained from frontline healthcare workers: 1 respiratory therapist (P100 RT 4-2020), 1 progressive care nurse (P100 RN 8MPLM 3-2020), and 1 medical intensive care nurse (P100 RN 8MICU 3-2020). The used cartridges were compared to an unopened and unused filter cartridge (P100- control) from the same SNS batch.

The cleaning protocol for the outer casing of the filters consists of wiping it down with premoistened quaternary ammonium/isopropyl alcohol wipes between patients. This procedure could be

\footnotetext{
Author for correspondence: Anthony Baffoe-Bonnie, E-mail: abaffoe6@vt.edu Cite this article: Baffoe-Bonnie AW, et al. (2022). Filtration evaluation of expired elastomeric P-100 filter cartridges after months of real-world use during the coronavirus disease 2019 (COVID-19) pandemic. Infection Control \& Hospital Epidemiology, 43: 824-825, https://doi.org/10.1017/ice.2021.104
}

repeated up to 10 times per day. At the end of the shift, the wiped filter pair is disassembled from the mask, which then undergoes further cleaning. ${ }^{4}$

There was no subjective feeling that the filters were difficult to breathe through by any of the users. No external damage apart from the expected "wear and tear" of the filter cartridge label, likely due to multiple episodes of wiping and cleaning, was noted with the used filters.

Filtration efficiency of the used filters and the control P100 filters from our expired stock was quantified using the methodology described by Patolia et al. ${ }^{5}$ The pair of filter cartridges from each user was tested with the testing run in duplicate.

The filtration efficiencies of the control, RT 4-2020, and RN 8MPLM 3-2020 cartridges were mostly $>95 \%$ across different particle sizes. The filtration efficiency of the P-100 RN 8MICU 3-2020 cartridge was $\sim 90 \%-95 \%$ but was not significantly different from that of the control (Fig. 1).

Elastomeric filters that were in good condition but long past their shelf life remained effective at filtering out aerosol-sized particles after being used in the "real-world" healthcare setting. We saw a small but nonsignificant decrease in filter efficiency in 1 of the 2 filter pairs that had been in use for 6 months. Our findings suggest that similar SNS supplies of well-kept but expired P100 filters may be used in the healthcare setting for at least 6 months, in contrast to other published literature regarding the duration of use of filter cartridges. ${ }^{2,3}$

The quantitative data demonstrated in our study is a strength, although the small sample size is a limitation. Tests were performed in duplicate to address this issue. We were unable to test the same exact filter cartridges before and after use because the testing process renders the filter device unusable. We caution that these findings may not be applicable to nonexpired filters, but we intuitively suspect that the duration of use could be longer.

With the continued coronavirus disease 2019 (COVID-19) pandemic and the possible surge in recently described highly transmissible variants, we anticipate that the use of elastomeric respirators will remain a key component in HCW protection. 